\title{
Knowledge management for radioactive waste management organisations
}

\section{P. Gowin*}

International Project on Innovative Nuclear Reactors

and Fuel Cycles (INPRO)

International Atomic Energy Agency

P.O. Box 100A-1400 Vienna, Austria

E-mail: p.gowin@iaea.org

*Corresponding author

\section{J. Kinker}

Waste Technology Section

Division of Fuel Cycle and Waste

Nuclear Energy Department of IAEA

P.O. Box 100A-1400 Vienna, Austria

E-mail: j.kinker@iaea.org

\section{A. Kosilov}

NENP - Nuclear Power Engineering Section

International Atomic Energy Agency

P.O. Box 100A-1400 Vienna, Austria

E-mail: a.kosilov@iaea.org

\section{Upshall}

Waste and Nuclear Materials Department

Nuclear Decommissioning Authority

Curie Avenue, Didcot, Oxfordshire OX11 0RH, UK

E-mail: ian.upshall@nda.gov.uk

\section{Y. Yanev}

INIS \& Knowledge Management Section International Atomic Energy Agency P.O. Box 100A-1400 Vienna, Austria E-mail: y.yanev@iaea.org

\footnotetext{
Abstract: Starting in 2006, the International Atomic Energy Agency (IAEA) developed a guidance publication on knowledge management for radioactive waste management organisations. The publication is part of the IAEA Nuclear Energy Document Series at the level of Report. The purpose of the document was to provide guidance to the full range of decision makers (in both the public
} 
and private sectors) on planning, implementing and sustaining the critical nuclear and institutional knowledge needed for the safe and efficient management of radioactive waste. The guidance covers salient aspects of managing tacit, implicit and explicit knowledge, both in documented (record) form and as skills and experiences in human beings. It addresses information management, human resources, technical competence management, primary and continuing education, stakeholder involvement, management systems and approaches and knowledge analysis and integration.

Keywords: knowledge management; long term; radioactive waste knowledge; radioactive waste management; techniques; tools.

Reference to this paper should be made as follows: Gowin, P., Kinker, J., Kosilov, A., Upshall, I. and Yanev, Y. (2009) 'Knowledge management for radioactive waste management organisations', Int. J. Nuclear Knowledge Management, Vol. 3, No. 2, pp.157-169.

Biographical notes: Peter J. Gowin is working with the International Atomic Energy Agency (IAEA) as a Programme Liaison Officer of the International Project on Innovative Nuclear Reactors and Fuel Cycles (INPRO). He joined the IAEA in 1997 and has held various positions in the Department of Nuclear Energy. Prior to joining the IAEA, he worked for German energy utility from Energie Baden-Württemberg. He studied in Vienna, Stuttgart and Oxford and holds a Master's in Physics and a PhD in Energy Economics.

John Kinker obtained a BSc in Environmental Engineering from the New Mexico Institute of Mining and Technology, USA, in 1989. He has worked as a Consulting Engineer in hazardous and radioactive waste management, treatment, storage and disposal facility decommissioning, modelling and engineering design analysis, nuclear facility safety and hazards analysis, nonreactor nuclear facility operations, hazardous waste site remediation, analytical quality assurance, statistical analysis, wastewater treatment design, groundwater modelling and extraction system design, site characterisation management and field experience in drilling and sampling of contaminated environmental media. He has worked primarily in the area of radioactive and mixed waste management (including work for the Los Alamos National Labs and Sandia National Laboratory) and performed similar work for major commercial clients such as British Petroleum and Westinghouse Nuclear Fuels. $\mathrm{He}$ is currently the Scientific Secretary for Waste Management Information Systems at the IAEA.

Andrei Kosilov obtained his $\mathrm{PhD}$ in Nuclear Engineering at the Moscow Institute for Physics and Engineering (MIPhI), Russia. His particular areas of experience include knowledge management for nuclear industry, nuclear education, human resources development, configuration management, SAT-based training for NPP personnel, management of training document development, NPP instrumentation and control, human-machine interface issues, operator support systems for NPPs, control room systems design, computerisation of operations and maintenance of NPPs and development of web applications. His curriculum vitae contains around 80 publications in national and international journals or presented at international conferences; more than 40 IAEA technical reports have been developed under his supervision. In 2001, he received the American Nuclear Society Training Excellence Award. 
Ian Upshall is the Information and Data Recording Manager at the Harwell office of the UK's Nuclear Decommissioning Authority (NDA), the organisation responsible for managing the civil nuclear liabilities and development of the proposed deep geological waste disposal facility. He has a degree in Applied Physics with Mathematics (Bournemouth). He joined the UK Atomic Energy Authority (UKAEA) in 1978 as a Scientific Officer working on a $100 \mathrm{MW}(\mathrm{e})$ prototype heavy water reactor. His involvement in nuclear materials accountancy and safeguards resulted in a transfer in 1991 to UKAEA Harwell as a member of the Nuclear Materials Accountancy and Control Team. He later became the Deputy Manager of the UK Safeguards Support Programme to the IAEA. In 2000, he moved to UK Nirex Ltd., also based at Harwell, which was responsible for the review of proposals for waste package disposability and development of the deep geological waste disposal facility.

Yanko Yanev studied at the University of Sofia, Bulgaria, obtaining an MSc in Nuclear Chemistry and a $\mathrm{PhD}$ in Environmental Radiochemistry. For more than 20 years, he developed an academic career as a Professor of Nuclear Chemistry of the University of Sofia. In 1991, he was appointed Chairman of a government commission which reviewed the nuclear power industry in Bulgaria. Furthermore, he was the President of the State Committee on the Use of Atomic Energy for Peaceful Purposes from 1991 to 1996 (the Bulgarian Atomic Energy Commission) and Vice Chairman of the IAEA's Board of Governors. He has acted as a Senior Energy Consultant to the Bulgarian-Russian Investment Bank and took up the post at the Department of Nuclear Energy of the IAEA in 1998. In 2001, he was appointed Section Head of the International Nuclear Information System (INIS) and the head of Nuclear Knowledge Management Unit since 2002.

\section{Introduction}

The management of radioactive waste is a long-term commitment comprising a number of stages, including: waste creation; pre-treatment; treatment; conditioning; storage; transportation; and disposal. These stages may vary from country to country. Nevertheless, they are representative of the stages in a generic radioactive waste life cycle.

There is a risk that a decision based on incomplete knowledge in one stage will have the potential to foreclose an option in a subsequent stage. Knowledge management will help identify where there are long-term interdependencies between the planning, designing, constructing, operating and decommissioning phases of a radioactive waste management facility.

Starting in 2006, the International Atomic Energy Agency (IAEA) developed the guidance publication on Knowledge Management for Radioactive Waste Management Organizations. ${ }^{1}$ The publication is part of the IAEA Nuclear Energy Document Series at the level of Report.

The purpose of the document was to provide guidance to the full range of decision makers (in both the public and private sectors) on planning, implementing and sustaining critical nuclear and institutional knowledge needed for the safe and efficient management of radioactive waste. The guidance covers salient aspects of managing tacit, implicit and explicit knowledge, both in documented (record) form and as skills and experiences 
in human beings. It addresses information management, human resources, technical competence management, primary and continuing education, stakeholder involvement, management systems and approaches, and knowledge analysis and integration.

\section{Challenges for managing nuclear knowledge in radioactive waste management organisations}

It is now generally accepted that the fundamental objective of radioactive waste management is to deal with the waste in a manner that protects human health and the environment now and in the future without imposing undue burdens on future generations. This objective first appeared in a 1995 IAEA Safety Series document (IAEA, 1995). The waste management principles that underpinned this objective have now been incorporated in a new generic Safety Standard and a review of the ten new principles clearly demonstrates the need to adopt a working culture that is largely dependant on access to information and knowledge. Effective knowledge management can therefore have a very positive effect on maintaining and improving safety in our waste management activities.

Knowledge management in a radioactive waste management environment requires specifically defined processes and a framework that must be organised to support the planning recording, dissemination and transfer of information (collectively 'management'). Knowledge management in radioactive waste management operations (and their regulation) is primarily concerned with ensuring that present and future generations of planners, decision makers and operators have access to appropriate sources of information and that the knowledge created can be efficiently and accurately disseminated and transferred. This must be done in such a way that key personnel are equipped with the necessary skills and competencies to understand and use the knowledge. The ultimate concern must be to support the long-term safe, efficient and cost effective management of the radioactive waste management facilities and their contents.

Knowledge management will also make a major contribution to the retention and retrieval of information over the long periods of time peculiar to radioactive waste management. Retention of critical staff and knowledge transfer to new staff will clearly be necessary - this may be achieved through succession planning, training, mentoring, and other knowledge transfer processes.

Among the key benefits for implementing knowledge management as an integral part of the business and management culture of a radioactive waste management facility are: safety; operating efficiency; and economic efficiency.

Of the three key driving forces for implementing knowledge management in radioactive waste management programmes, the most important stems from operational safety and long-term stewardship. Every treatment, storage and disposal facility operator must ensure that critical knowledge and skills related to safe operation are appropriately gained, maintained and transferred during the facility lifetime. This may span several decades for treatment and storage facilities and several centuries for disposal facilities. Knowledge management is not a generic application that can be 'installed' and implemented - the techniques used will be different depending on the facility, the organisation and Member State. The developers, operators and regulators of these facilities have specific knowledge requirements for meeting their respective responsibilities and these will largely determine the most effective techniques. 
In addition to safe operation, radioactive waste management organisations are likely to be concerned with operational efficiency (or optimisation). Where this is the case the motivation for adopting knowledge management could be different. However, safety and operational efficiency can be complementary, based upon a skilled and sustained workforce - a serious challenge when considering the timescales relating to radioactive waste management. Loss of skills and knowledge resulting from an increasingly mobile workforce, in addition to natural wastage, is a particular concern.

Increasingly, government funded waste management organisations have to demonstrate that they are investing public funds in an effective way. In commercial organisations, the economic effectiveness of an organisation is likely to be a significant factor in its long term viability. Thus, having a knowledgeable workforce that is able to make justifiable and well founded decisions can have an impact on economic efficiency.

Regulatory organisations need to be assured that key decisions are made on sound bases and that the outcomes are accurately transmitted and understood. Communicating the basis for a decision can be very important when it is considered that some will have an impact on future generations living and operating in a significantly different regulatory environment. This suggests that records containing the legislation of the day must be retained in order to enable a future society to interpret and understand the basis for historical decisions.

Facilities dealing with radioactive waste include the integration of two primary types of knowledge: about the facility itself; and about the waste and any associated processes carried out.

These two knowledge streams, and the associated documented information, will together represent the bulk of the input used in the decision-making process. For example, there will be many factors that will influence the evolving management strategy during the period that the waste is subject to active management. These will include revised or updated technical data, changes to regulation, environmental considerations and so on, some of which are associated with the waste and some on the performance of the facility. Therefore, knowledge associated with all potential management strategies should be retained - even if there is a low possibility that they will be adopted. If new information brings into question the validity or appropriateness of an historical decision, national policy may require that it is necessary to review the basis of the decision in terms of the effect of contemporary knowledge.

Controlling potential long-term liability also requires knowledge to be preserved. Preserving the basis for decisions, for example, can protect a radioactive waste management facility operator and its legal successors over a long period of time against charges of negligence or capriciousness in making safety related decisions. This also protects the legal interest of the public and the government regulatory organisation.

The report ${ }^{1}$ outlines the challenges in managing nuclear knowledge that are specific to radioactive waste management organisations. They are:

Challenge 1 Need to consider long timescales

Challenge 2 Operating waste management facilities with limited experience

Challenge 3 Integrating knowledge from multiple sources

Challenge 4 Need to involve many stakeholders

Challenge 5 Need for education and training in radioactive waste management. 


\section{Managing nuclear knowledge over very long time scales}

Management time scales will vary depending on the disposal facility and the waste type. In order to illustrate typical time scales, Table 1 shows the differences between High Level Waste (HLW) including spent fuel and Long-Lived (LL) Low and Intermediate Level Wastes (LILW), Short-Lived (SL) LILW, and Very Low Level Waste (VLLW) (these waste types are not uniformly defined or recognised by Member States - they are used here simply to aid comparison).

Table 1 Typical time scales for aspects of various types of disposal systems/repositories

\begin{tabular}{|c|c|c|c|}
\hline Waste type & $\begin{array}{l}\text { HLW/Long Lived } \\
\text { Intermediate Level } \\
\text { Waste }(\text { LL-LILW) }\end{array}$ & $\begin{array}{l}\text { Short Lived Low and } \\
\text { Intermediate Level Waste } \\
(\text { SL-LILW) }\end{array}$ & $\begin{array}{l}\text { Very Low Level Waste } \\
(\text { VLLW) }\end{array}$ \\
\hline $\begin{array}{l}\text { Type of } \\
\text { disposal system }\end{array}$ & Geological repository & $\begin{array}{l}\text { Near surface } \\
\text { burial facility }\end{array}$ & $\begin{array}{l}\text { Near surface } \\
\text { burial facility }\end{array}$ \\
\hline $\begin{array}{l}\text { Implementation } \\
\text { time }\end{array}$ & $>100$ years & $\begin{array}{l}\text { Several decades } \\
\text { (typically) }\end{array}$ & $\begin{array}{l}\text { Several decades } \\
\text { (typically) }\end{array}$ \\
\hline $\begin{array}{l}\text { Duration of } \\
\text { potential risk }\end{array}$ & $\begin{array}{l}\text { Several thousand to } \\
\sim \text { one million years }\end{array}$ & 100 to 300 years & A few decades \\
\hline Status & $\begin{array}{l}\text { Prospective: under } \\
\text { development }\end{array}$ & Ongoing experience & Ongoing experience \\
\hline
\end{tabular}

It is necessary to consider that one cannot make judgements about the evolution of, for example, technology, knowledge, regulatory requirements, the stability of political interest or human society. Therefore, the principles underpinning the knowledge management system must be such that systems can be implemented to capture and process the knowledge necessary for the safe and effective operation of facilities over the various phases, including that of post-closure.

The very long timeframe involved in the HLW disposal facility life cycle requires a phased or step-by-step decision-making approach to developing, managing and closing such a repository. This suggests that:

- $\quad$ knowledge must be continuously validated for long-term waste disposal, whilst, in contrast, continuous assessment and improvement would be the best approach for managing SL-LILW disposal facilities (ongoing process with a number of facilities in various Member States with the sharing of lessons learned).

- $\quad$ the knowledge management system for a HLW disposal facility needs to be evaluated in terms of its relation or potential importance to the main goals and life cycles of the radioactive waste management system ('grading' its importance to safety is a common approach to help allocate resources most effectively).

\subsection{Knowledge about radioactive waste in perspective}

Information about radioactive waste is created in all phases of its management - from its generation, through processing, storage and transportation, disposal and post-facility closure. It is not known how long it will be necessary to undertake extended environmental monitoring and disposal facility performance evaluation but it could amount to many decades. 
Systems for the disposal of radioactive waste must meet a set of minimum standards for the long-term protection of human health and the environment. These standards may evolve over time and the systems must be suitably flexible and robust to provide the necessary assurances to society. The IAEA's implementation guidance for its HLW repository safety standards, ${ }^{2}$ states that the application of a long-term monitoring programme would be a reasonable societal expectation as long as there are appropriate institutions to perform the task. This post-closure monitoring will require that adequate knowledge about disposal sites is made available for as long as these programmes are in place.

The knowledge management arrangements are driven not just by the immediate needs of the present waste custodian but also to whom responsibility falls in the future. The relevance of the information passed on may become less obvious as time goes by, therefore it becomes increasingly important to also transfer contextual information:

\begin{abstract}
"To make it possible for future generations to read, understand and interpret the information, it will be important to retain and transmit contextual information (for example, policy for regulating waste, rationale for safety arguments and choices, language and technical terminology, scientific understanding, methods for collecting, analysing and interpreting measurements) as well as the actual recorded data.",
\end{abstract}

This ongoing requirement means that radioactive waste managers need to work with each other, and their licensing and regulatory authorities, to ensure that the right information is created and maintained at each appropriate step along the way.

\title{
3.2 Knowledge at various stages of radioactive waste management
}

The major sources of information are the waste generator and the waste conditioner/packager. Waste generation - and consequently the generation of important information - has typically preceded the start of planned waste management programmes. Whilst there is a certain amount of historical information to be managed all future activities should recognise the information management needs. For the waste management knowledge management system it is essential to ensure that the information acquisition, managing and processing to support the generation of adequate knowledge also supports its transfer for use in the next stage of the waste life cycle and/or for the next generation of managers and workers (IAEA, 2004). ${ }^{1}$

\section{Implementing knowledge management in radioactive waste management organisations}

Knowledge management is a business management concept which relies heavily on human resources, information technology and process. Therefore, knowledge management should leverage, and be integrated into, an organisation's culture, business processes, management strategy, operational structures, information technology, and stakeholder relationships. This means that any knowledge management strategy should be part of the corporate strategic and policy documents of the organisation and receive clear commitment from the management team. The degree of success in adopting knowledge management should be measured against staff-based and organisational 
performance metrics. Particular processes and tools that bring benefits through knowledge sharing should be integrated into the business culture and social and intellectual networks where these have an impact on radioactive waste management.

Knowledge is, by definition, multidisciplinary and this characteristic is particularly apparent in the field of radioactive waste management. Where the purpose of specific knowledge management processes are not generally recognised for what they are, it may be necessary to highlight and tailor them and to phase them in over a period of time. The IAEA report ${ }^{1}$ provides guidelines for phasing and structuring knowledge management practices into an existing and functioning radioactive waste management facility, including:

- the assessment of existing knowledge management practices and knowledge inventory analysis

- the development of a viable strategy that includes the assignment of roles and responsibilities

- strategies for knowledge management contingency plans

- performance measures of knowledge management success at both staff and organisational level.

Ultimately, the benefits of knowledge management will only be realised when the practices become an integral part of the facility's everyday operating ethos. Success relies on staff to think from a knowledge management perspective when designing workflows and to look beyond performing just the task at hand to considering how knowledge can be captured and shared both now and in the future. This fundamental change in perspective will require top-level support from senior managers in the form of clear expectations, adequate resources, and rewards for desired behaviours and results.

Implementing an effective, knowledge management strategy may require some fundamental changes to the facility's organisational culture. Although some benefits can be realised on a short timescale, major institutional changes of this nature can take several years to bed in. Because of the significance and duration of the change process, active change management is essential to the success of knowledge management. Managing the changes will require clear communications, top-down and bottom-up alignment, building momentum on early successes, and most importantly, leadership to direct the effort over the long-term.

\subsection{Organisational obligations}

In an ideal implementation, all organisations forming the radioactive waste community with responsibilities for information preservation, transfer and knowledge creation would incorporate a fully compliant, standards-based integrated framework as the basis of their information management system.

Specific organisational obligations would include:

- the creation and preservation of appropriate information resources in accordance with relevant standards

- the management of those resources in a system that documents and preserves the context of their creation and use 
- making available to the wider waste management community the information surrogates representing context entities in a form suitable for citation, linking and data exchange

- $\quad$ linking to the relevant context surrogates in other sites or clusters in the broader radioactive waste information network

- the documentation of their information resource management system as part of quality management system processes

- responsibility for the quality of the data and information in their own systems

- the sharing of any knowledge, technologies or systems they develop to facilitate the utilisation of contextual information

- financing this activity as a part of standard operational practice.

Bodies that currently have responsibilities for the standards, protocols and training programmes that support the radioactive waste community should take on the responsibility for developing and managing such programmes that may be required to sustain the utilisation of integrated frameworks over time.

\subsection{Implementation}

If the radioactive waste community or any part of it decided to implement an integrated framework, their first task would be the development of an implementation strategy. The issues that such a strategy should consider include:

- $\quad$ setting up a pilot project to evaluate the approach and develop a detailed implementation strategy

- $\quad$ assessing the existing information resource management systems and planning their upgrade to include the required capabilities. Particular attention to be paid to archival, record management and knowledge management systems

- defining the purpose and limits of the integrated framework. These can be scaled or graded to meet particular needs and circumstances as required

- $\quad$ gearing the contextual information system to work with existing information systems as far as possible without compromising functionality

- reusing existing contextual information resources wherever possible to minimise duplication of effort

- $\quad$ identifying and utilising the appropriate open source or non-proprietary contextual information management tools

- involving all staff concerned with the management of information, in particular the officer(s) responsible for the management system, knowledge managers, archivists, records managers, librarians, and information officers

- $\quad$ training of staff, including senior management, and defining new tasks and job descriptions 
- $\quad$ maintaining the hardware and software to support the system, including backup strategies

- $\quad$ establishing information quality review processes.

\subsection{Benefits and risks}

In addition to the generic benefits that stem from information managed in an open network systems, it is possible to identify specific benefits that would arise from the implementation of the contextual information frameworks across the radioactive waste management community. These would include:

- facilitating the capture of critical implicit technical knowledge, mutual learning, and the sharing of experience both within organisations and between organisations

- $\quad$ utilising existing technical and operational knowledge and information that has already been accumulated and therefore building on existing resources in a structured way

- $\quad$ enabling all stakeholders to understand the bigger picture, thus promoting trust and confidence in the community

- complementing existing practice as it is a non-invasive technique that builds frameworks that will enable much more to be done with existing information

- helping avoid contradictory decisions being taken in the future and improve decision making more generally

- $\quad$ enabling the informed appraisal and selection of information resources for future needs based on shared experience and information

- enabling the informed, confident, systematic, responsible and registered destruction of information resources no longer deemed to be of value

- $\quad$ providing a quality feedback mechanism

- $\quad$ improving transparency both within the industry and to the wider community

- facilitating indirect review of information content by regulatory and other appropriate external organisations.

However, all strategies and technologies have inherent risks that need to be considered and evaluated. The risks to such an approach could include:

- $\quad$ Concern in the radioactive waste industry about the use of an open network to reference potentially sensitive information about radioactive materials. Each organisation responsible for a contextual information framework would decide what primary information should be placed in the public domain. Much information that is required to make the framework effective is already in the public domain but not systematically managed. As time passes the security implications associated with much of the information are likely to reduce as they become of historic rather than operation value. 
- Concerns about the longevity of electronic information technologies. It is indeed the case that the concept makes use of contemporary technologies and expertise and it is recognised that there is nervousness about using the digital technologies and digital networks. However, critical information should be replicated in other systems using different technologies or media and this applies equally to contextual information.

- Concerns that such strategies will lead to increased costs and increased work loads. There is no question that this strategy will require adequate resourcing. It is contended that as the strategy makes better use of information that already exists and that the system, once in place, will add significantly to administrative and governance productivity, and thus reduce costs over time.

- Concerns about unintended consequences. All new activities and technologies run the risk of experiencing unintended consequences. Web-based and networked information system technologies are in their infancy and their impact on society is only just becoming apparent. The adoption of the approach should be undertaken in a measured and self-reflective manner to ensure that they are not counterproductive.

There are also risks associated with the failure to implement an integrated information management strategy. The decision not to use this approach would, by default, leave the industry to continue to adopt unsustainable practices which have been shown to be inadequate. Indeed, it could be argued that the industry could be seen to be negligent if it failed to implement strategies that have been codified by international archival and records management standards.

More specifically these risks could include:

- $\quad$ increasing the degree of burden on future generations

- $\quad$ criticism that the industry is not adequately addressing its responsibilities

- $\quad$ increasing the likelihood of accidents

- criticism for not using best practice tools and concepts

- loss of critical information through poorly managed organisational change

- duplication of work and inefficient use of resources

- loss of implicit knowledge

- $\quad$ increasing the likelihood of inaccurate information having a misleading influence on community attitudes and political decision making.

\section{Knowledge management tools and techniques for use in radioactive waste management}

Knowledge management in radioactive waste management processes, whether operational or regulatory, is just now being established. Therefore, the tools and techniques discussed here are taken from other applications in which knowledge management has been successfully established. It is suggested that the general principles 
can be adapted to the meet the particular aspects of waste management operations. In time, the experience gained will allow a review of the effectiveness of these techniques in a radioactive waste management environment.

As for these other domains that already apply knowledge management techniques, it is require to adopt robust planning and well-defined management processes within a business framework. The planning must involve all stakeholders, including knowledge owners and producers as well as knowledge users. The process must be organised to support the alignment, sharing, transfer and management of human resources and information related to radioactive waste management. This must be done in such a way that the people have, not only access to information, but the skills and competencies to understand and use it. The knowledge bases and the existing information must be appropriate to support a safe, efficient and cost effective operation of the waste management facilities and their contents. Given that these facilities often have long life cycles, it is important to maintain the knowledge for a long period of time. It is equally important that the life time plan is supported by effective staff succession planning, talent retention and competence management.

The report ${ }^{1}$ lays out the aspects of knowledge management that may be usefully adapted for use in the management of radioactive waste. It includes discussions about knowledge-maintenance infrastructures as well as talent management and human resources.

\section{Conclusions}

Over the past years, it has become increasingly clear that managing nuclear knowledge is of prime importance for any nuclear endeavour. Good nuclear knowledge management programmes can - in most general terms - contribute to:

- achieving safe operation and maintenance of all nuclear facilities

- achieving gains in economics and operational performance through effective management of the resource knowledge

- facilitating innovation to achieve significant improvements in the safe, economical operation of all new nuclear projects

- maximising the flow of nuclear knowledge from one generation to the next and to attract, maintain, and further develop a dedicated cadre of highly competent professional staff to sustain nuclear competence

- achieving a high degree of transparency - regional, national, and international - and exchange of operational experience to ensure nuclear safety while simultaneously achieving appropriate safeguard requirements by avoiding the improper use of sensitive information.

The IAEA report ${ }^{1}$ recommends that nuclear knowledge management programmes should be established also for radioactive waste management, both on the level of radioactive waste management organisations as well as possibly also as part of related national radioactive waste management programmes. 
For doing so, the IAEA has already published a number of documents applicable to nuclear organisations in general. In particular IAEA-TECDOC-1510 on Knowledge Management for Nuclear Industry Operating Organisations (IAEA, 2006) is applicable also to radioactive waste management organisations, contains relevant guidance and should be read together with this publication. Establishing and implementing nuclear knowledge management programmes in the context of radioactive waste management poses special challenges, though. The report ${ }^{1}$ is meant as additional guidance for addressing these challenges, which are:

- $\quad$ the long timescales to be considered

- the need to run programmes based on limited experience gained to-date

- the need to integrated relevant knowledge from different sources

- the desired involvement of stakeholders

- the need to develop adequate education and training programmes for radioactive waste management.

It is written to be specific for nuclear knowledge management in a radioactive waste management context, and provides strategic advice to managers on how to embark on and implement nuclear knowledge management programmes in a radioactive waste management context.

\section{References}

International Atomic Energy Agency (IAEA) (1995) 'The principles of radioactive waste management', Safety Series No. 111-F, IAEA, Vienna.

International Atomic Energy Agency (IAEA) (2004) Records for Radioactive Waste Management Up to Repository Closure: Managing the Primary Level Information (PLI) Set, IAEA-TECDOC-1398, IAEA, Vienna.

International Atomic Energy Agency (IAEA) (2006) Knowledge Management for Nuclear Industry Operating Organizations, IAEA-TECDOC-1510, IAEA, Vienna.

\section{Notes}

1 International Atomic Energy Agency (IAEA), Knowledge Management for Radioactive Waste Management Organizations, Vienna (in preparation).

2 International Atomic Energy Agency (IAEA), Geological Disposal of Radioactive Waste, IAEA Safety Guide (in preparation).

3 International Atomic Energy Agency (IAEA), Management System for Geological Disposal of Radioactive Waste, IAEA Safety Requirement (in preparation). 